\title{
Lipidic Profiles of Patients Starting Peritoneal Dialysis Suggest an Increased Cardiovascular Risk Beyond Classical Dyslipidemia Biomarkers
}

Julia Hernández Lluesa

Health Research Institute Hospital La Fe (IIS La Fe)

Luis Carlos López-Romero ( $\square$ luiscarloslopezromero@gmail.com )

Hospital Universitari i Politècnic La Fe

José Jesús Broseta

Hospital Clínic of Barcelona

Marta Roca Marugán

Health Research Institute Hospital La Fe (IIS La Fe)

Iris Viejo Boyano

Hospital Universitari i Politècnic La Fe

Diana Rodríguez-Espinosa

Hospital Clínic of Barcelona

Aina Gómez-Bori

Hospital Universitari i Politècnic La Fe

Amparo Soldevila Orient

Hospital Universitari i Politècnic La Fe

Ramón Devesa-Such

Hospital Universitari i Politècnic La Fe

Pilar Sánchez Perez

Hospital Universitari i Politècnic La Fe

Julio Hernández-Jaras

Hospital Universitari i Politècnic La Fe

\section{Research Article}

Keywords: Lipidomics, Lipid profile, Peritoneal dialysis, Dyslipidemia

Posted Date: December 20th, 2021

DOI: https://doi.org/10.21203/rs.3.rs-1157347/v1 
License: (c) (i) This work is licensed under a Creative Commons Attribution 4.0 International License. Read Full License 


\section{Abstract}

Background. Lipids are molecules that constitute a fundamental part of the plasma. Chronic kidney disease (CKD) produces profound changes in lipid metabolism, and associated lipid disorders, in turn, contribute to the progression of CKD. Patients on peritoneal dialysis (PD) have more atherogenic lipid profiles than non-dialysis-dependent CKD patients.

Methods. Single-center prospective observational study of a cohort of CKD patients who started renal replacement therapy with continuous ambulatory peritoneal dialysis. The differences in the lipid profile and analytical variables before and six months after the start of peritoneal dialysis were analyzed. Samples were analyzed on an Ultra-Performance Liquid Chromatography system.

Results. Thirty-nine patients were enrolled in this study. Their mean age was $57.9 \pm 16.3$ years. A total of 157 endogenous lipid species of 11 lipid subclasses were identified. There were significant increases in total free fatty acids $(p<0.05)$, diacylglycerides $(p<0.01)$, triacylglycerides, $(p<0.01)$, phosphatidylcholines $(p<0.01)$, phosphatidylethanolamines $(p<0.01)$, ceramides $(p<0.01)$, sphingomyelins $(p<0.01)$, and cholesterol esters $(p<0.01)$ from baseline to 6 months. However, there were no differences in the classical lipid markers, neither lysophosphatidylcholines, monoacylglycerides, and sphingosine levels.

Conclusions. Patients on PD present changes in the lipidomic profile beyond the classic markers of dyslipidemia, that suggest an increased cardiovascular risk in them.

\section{Introduction}

Lipids are molecules that constitute a fundamental part of plasma and perform various cellular functions. They are mainly found as molecules of sterols, glycerophospholipids, fatty acids, and glycerolipids transported by lipoproteins (low-density lipoproteins [LDL] and high-density lipoproteins $[\mathrm{HDL}])^{1}$

Phospholipids $(\mathrm{PL})$ are known to be a structural part of cell membranes, perform metabolic functions and contain a variety of fatty acids such as phosphatidylserine (PS), phosphatidylethanolamine (PE), phosphatidylcholine (PC), phosphatidylinositol (PI), lysophosphatidylcholine (lysoPC) and sphingomyelin $(\mathrm{SM})^{2}$. Only triglycerides (TG), LDL, HDL, and total cholesterol (TC) levels are measured in routine clinical practice as biomarkers of lipid metabolism associated with cardiovascular risk.

Patients with chronic kidney disease (CKD) have an increased risk of cardiovascular disease (CVD), to such extent that they account for most of the mortality and morbidity causes observed in this population ${ }^{3}$. Many other conventional risk factors for cardiovascular disease are present in patients with CKD, such as hypertension, insulin resistance, as well as underlying conditions like chronic inflammation, endothelial dysfunction, and arterial stiffness ${ }^{4}$. It is known that CKD produces profound changes in lipid 
metabolism, and these associated lipid disorders, in turn, contribute to the progression of CKD and its cardiovascular complications ${ }^{5,6}$.

Some authors suggest that patients on peritoneal dialysis (PD) have a more atherogenic lipid profile than non-dialysis-dependent CKD (NDD-CKD) patients or on hemodialysis (HD) ${ }^{7}$. This atherogenic profile is generated both by the loss of proteins in the dialysate and by the use of high glucose-based solutions ${ }^{8}$. Furthermore, exposure to high glucose dialysis solutions may potentially accelerate atherosclerosis and stimulate cytokines that promote chronic inflammation ${ }^{9}$. In the general population, the reduction of total cholesterol and LDL levels is associated with improved clinical outcomes in terms of morbidity and mortality; however, several studies and randomized clinical trials have failed to obtain these same results in patients on dialysis despite receiving treatment with lipid-lowering drugs and achieving significant reductions in $L D L, C T$, and TG levels ${ }^{10,11}$.

In recent years, the development of new technologies has produced advances in the study of the lipid profile. Lipidomics is a branch of metabolomics and a newly emerged discipline whose function is to characterize and quantify lipid molecular species and their biological role in expressing proteins involved in lipid metabolism and function ${ }^{12}$. It has been widely applied in disease biomarkers discovery ${ }^{13}$, disease mechanism research ${ }^{14}$, and drug development ${ }^{15}$. The application of these tools has demonstrated that the metabolism of phospholipids and fatty acids is modified in the context of CKD ${ }^{16}$.

Some metabolic networks are essential to most lipids, including sphingolipids, glycerophospholipids, and non-esterified fatty acids (NEFA). Understanding these pathways and networks will allow interpreting how and why lipids change after a specific physiological perturbation. Proton nuclear magnetic resonance spectroscopy (PNMR) and liquid chromatography-mass spectrometry (LC-MS)-based metabolomics techniques have been applied to determine CKD's metabolic features ${ }^{17}$. The use of lipidomics to detect a broader panel of lipid species can improve the prediction of the different alterations that influence cardiovascular mortality and the ultra-performance liquid chromatography-quadrupole time-of-flight highdefinition mass spectrometry (UPLC-QTOF) is regarded as one of the best analytical tools for this purpose $^{18}$.

Most of the numerous clinical studies focused mainly on studying classic species of lipids; however, studies of the global lipid profile in this group of patients are highly needed to comprehensively evaluate the effects in patients with end-stage chronic kidney disease.

This study aimed to analyze the lipid profile of PD patients using liquid chromatography and highresolution mass spectrometry at the beginning and six months after the start of the technique.

\section{Methods}

\section{Study design}


This is a single-center prospective observational study of a cohort of CKD patients who start renal replacement therapy with continuous ambulatory peritoneal dialysis (CAPD). This study was performed according to the ethical principles of the Declaration of Helsinki. Written informed consent was obtained from all participants before enrollment. This study is part of the project "Application of proteomics and/or metabolomics techniques for the identification and comparative analysis of uremic solutes," approved by Hospital Universitari i Politècnic La Fe Clinical Research Ethics Committee with reference number 2014/0836.

\section{Study population}

Data from 39 patients followed by the Department of Nephrology of Hospital Universitari i Politècnic La Fe from December 2016 to September 2018 were examined.

Subjects older than 18 years with end-stage CKD who had been seen in the clinic for at least six months and were programmed to start CAPD were included. Patients with malignant diseases, history of peritonitis, on treatment expecting renal function recovery, hospitalization in the previous three months, transferred from chronic HD, or failed kidney transplantation were excluded. The PD prescription was left at the attending physician's discretion according to the patients' depurative and ultrafiltration requirements. All patients received commercially available glucose-based (1.36\% and $2.27 \%)$ DP Dianeal solutions, and Y-sets, and twin-bag systems were applied in all cases.

\section{Data and laboratories collection}

Baseline demographics including age, gender, major comorbidities, etiology of kidney disease, statin use, and clinical data were collected from medical records at the initiation of PD. All samples for routine laboratories and lipidomics studies were obtained the day before starting PD (T0) and six months after (T1). Blood samples were collected from the patients after an overnight fast and were processed immediately after sampling. Laboratory parameters included creatinine, estimated glomerular filtration rate (eGFR), glucose, TC, TG, HDL, and LDL were determined in the hospital biochemistry laboratory by assays following Good Laboratory Practice standards. Following the unit's usual clinical practice, a peritoneal equilibration test (PET) was scheduled on T1.

The KDIGO guidelines were adopted to evaluate and manage dyslipidemia; thus, patients who were not receiving lipid-lowering therapy before starting the study did not begin statin therapy when starting CAPD $^{19}$.

\section{Lipidomics analysis}

\section{Sample preparation for mass spectrometry}

Lipids were extracted by adding $150 \mu \mathrm{L}$ of cold isopropanol to each dried sample, vortexed, and kept at $-20^{\circ} \mathrm{C}$ for $30 \mathrm{~min}$ for protein precipitation. After centrifugation at $13000 \mathrm{~g}\left(10 \mathrm{~min}, 4^{\circ} \mathrm{C}\right), 20 \mu \mathrm{L}$ of the supernatant were transferred to a $96-$ well plate and $10 \mu \mathrm{L}$ of an internal standard mix solution (IS, $20 \mu \mathrm{M})$, 
containing lipids from different classes, were added to each sample. The mixture was finally diluted with $70 \mu \mathrm{L}$ of Mobile phase A. A quality control (QC) sample was prepared by mixing $10 \mu \mathrm{L}$ of each sample. A blank was prepared to identify potential artifacts and analyzed at the end of the analytical sequence. Finally, samples were randomly injected into the chromatographic system in order to avoid intra-batch variability, as well as to enhance quality and reproducibility. One QC Sample was injected every five samples. Stability and analytical drift were investigated through IS intensities.

\section{UPLC-QToF analysis}

Samples were analyzed on an Ultra-Performance Liquid Chromatography (UPLC) system coupled to an iFunnel quadrupole time of flight (QTOF) Agilent 6550 spectrometer (Agilent Technologies, CA, USA). The chromatographic separation of lipids was carried out using an Acquity UPLC C18 CSH chromatographic column (100 x $2.1 \mathrm{~mm}, 1.8 \mu \mathrm{m})$ from Waters (Wexford, Milford, MA, USA). The UPLC-MS method employed was previously described by Alcoriza et $\mathrm{al}^{20}$. Briefly, for $\mathrm{ESI}(+)$ mode, the mobile phases consisted of (A) $10 \mathrm{mM}$ ammonium formate in 60:40 (v/v) acetonitrile:water and (B) $10 \mathrm{mM}$ ammonium formate in 90:10 (v/v) isopropanol:acetonitrile and a flow rate of $0.4 \mathrm{ml} / \mathrm{min}$; for ESI(-) mode, ammonium acetate was used as modifier, and the flow rate employed was $0.6 \mathrm{ml} / \mathrm{min}$. Autosampler and column temperatures were set to $4^{\circ} \mathrm{C}$ and $65^{\circ} \mathrm{C}$, respectively, and the injection volume was $5 \mu$.

Samples and QC were acquired using Full scan MS data from 50 to $1700 \mathrm{~m} / \mathrm{z}$ with a scan frequency of 6 Hz. QC were also acquired using dependent data acquisition (DDA), by Auto MS/MS mode, and dataindependent acquisition (DIA), by using the all-ion fragmentation mode, both using 0 and $40 \mathrm{~V}$ as collision energies.

\section{Mass spectrometry data pre-processing and analysis}

Data processing of samples and QC acquired in Full MS Scan was done using an in-house R (v.3.6.1) processing script with XCMS and CAMERA packages for peak detection, noise filtering, and peak alignment. QC data acquired by DIA mode were processed and annotated using the LipidMS package published by Alcoriza et al. ${ }^{20}$. Parameters selected for peak peaking and peak grouping were based on previous instrumental data analysis experience. The resulting data matrix was generated, including annotated molecular features, sample ID (observations), and peak intensities. Finally, an internal standard-based normalization was performed and data filtered according to the quality assurance criteria of coefficient of variation $<30 \%$ in QC samples and the presence of the variable in $60 \%$ of the samples in at least one of the compared groups. The final dataset obtained with potential lipid identification was used for the statistical analysis.

\section{Statistical analysis}

The differences in the lipid profile analytical variables between baseline or T0 (before starting peritoneal dialysis) and T1 (6 months after the start of peritoneal dialysis) were analyzed. For the lipidomic analysis, each compound was grouped with its possible adducts and compound class based on the International Lipid Classification once the data table with the identifications had been obtained. 
Continuous variables were expressed as mean \pm standard deviation (SD), while categorical variables were expressed as percentages. The adjustment of the variables to a normal distribution was verified using the Kolmogorov-Smirnov test. The differences between the quantitative variables were analyzed using Student's t-test for paired data in cases of normally distributed variables and the Wilcoxon test for skewed data. Differences between individuals with different clinical variables were analyzed using Student's t-test for independent data or ANOVA.

A two-sided p-value inferior to 0.05 was considered statistically significant. Analyses were performed with IBM SPSS $®$ Statistics version 26 and graphics, with GraphPad version 9.

\section{Results}

Thirty-nine patients ( 26 men and 13 female) were enrolled the study. Every patient completed the six months follow-up. Their mean age was $57.9 \pm 16.3$ years. $71.8 \%$ of the patients were overweight or presented some degree of obesity with a mean body mass index of $26.72 \pm 4.12 \mathrm{Kg} / \mathrm{m}^{2}$. Underlying renal diseases were glomerular disease (15 patients), diabetic kidney disease (10), inherited kidney disease (4), nephroangiosclerosis (2), interstitial disease (2), and others (6). Hypertension was present in $96.3 \%$ of the patients, diabetes mellitus in $30.8 \%$, and 30 of the 39 patients had dyslipidemia (76.9\%). Of the group of patients with dyslipidaemia, only one of them was not on statin treatment.

Evolution of classic markers of dyslipidemia. Table 1 shows the results of the analytical variables of lipid profile (TC, LDL, HDL, and TG), serum glucose, and renal function before and six months after initiating renal replacement therapy with CAPD. The levels of CT and LDL were lower six months after CAPD initiation, but these were not statistically significant.

Table 1

Analytical variables at baseline (T0) and 6-month onset of CAPD (T1).

\begin{tabular}{|llll|}
\hline & T0 & T1 & P-value \\
\hline Creatinine & $5.22 \pm 1.06$ & $5.81 \pm 1.43$ & $<0.001$ \\
\hline eGFR (ml/min/1.73m2) & $10.78 \pm 2.13$ & $9.60 \pm 2.72$ & $<0.001$ \\
\hline Glucose & $96.13 \pm 25.86$ & $102.43 \pm 29.16$ & NS \\
\hline Total Cholesterol & $163.39 \pm 59.96$ & $156.91 \pm 40.61$ & NS \\
\hline LDL-C & $92.33 \pm 50.92$ & $84.19 \pm 34.20$ & NS \\
\hline HDL-C & $47.13 \pm 19.50$ & $45.08 \pm 10.57$ & NS \\
\hline Triglycerides & $137.17 \pm 79.33$ & $143.93 \pm 82.08$ & NS \\
\hline $\begin{array}{l}\text { eGFR, estimated glomerular filtration rate; } \text { LDL-C, low-density lipoprotein cholesterol; HDL, high- } \\
\text { density lipoprotein cholesterol; NS: Statistically non-significant. }\end{array}$ & \\
\hline
\end{tabular}


PETs were available from 39 patients. The mean D/P creatinine at 4 hours was $0.76 \pm 0.07$. The membrane transport type was high in $35.89 \%$ of the patients $(n=14)$, medium high in $51.28 \%(n=20)$, and medium-low in $12.83 \%(n=5)$.

\section{Results of the lipidomic study}

A total of 157 endogenous lipid species of 11 lipid subclasses were identified, including free fatty acids (FFA), monoacylglycerides (MAG), diacylglycerides (DAG), triacylglycerides (TAG), phosphatidylcholines (PC), lysophosphatidylcholines (LysoPC), ceramides (Cer), sphingomyelins (SM), sphingosine (SG) and cholesterol esters (CE). These results are summarized in Table 2.

Table 2

Lipid species identified by Ultra-Performance Liquid Chromatography (UPLC) system coupled to an iFunnel quadrupole time of flight (QTOF).

\begin{tabular}{|ll|}
\hline Lipid subclass & Identified compounds \\
\hline Free fatty acids & 26 \\
Glycerolipids & 3 \\
- Monoacylglycerides & 8 \\
- Diacylglycerides & 26 \\
- Triacylglycerides & \\
\hline Glycerophospholipids & 55 \\
- Phosphatidylcholine & 6 \\
- Lysophosphatidylcholine & 6 \\
- Phosphatidylethanolamide & \\
\hline Sphingolipids & 3 \\
- Ceramide & 1 \\
- Sphingosine & 19 \\
- Sphingomyelin & 4 \\
\hline Cholesterol esters & 157 \\
\hline Total & \\
\hline
\end{tabular}

To evaluate these findings, the majority adduct identified for each compound was selected. The differences between the two measurement times were evaluated by calculating the average data of the compounds of each group as total data. Using the plasma lipid profile, with a mass spectrometry approach, a significant increase $(p<0.05)$ in total free fatty acids was observed from T0 to T1. FFA $(16: 1)$ 
and FFA (18:0) were the FFAs with the highest peak response. In the glycerolipid group, diacylglycerides and triacylglycerides increased after initiating CAPD $(p<0.01)$. Monoacylglycerides had an increase but it was not statistically significant $(p=0.1)$. In the group of glycerophospholipids, the intensity peaks of phosphatidylcholines and phosphatidylethanolamines increased significantly $(p<0.01)$, but no significant changes were observed in the intensity peaks of lysophosphatidylcholines $(p=0.02)$. After evaluating the response of sphingolipids, we found that the response peaks of ceramides and sphingomyelins experienced a significant increase at T1 $(p<0.01)$. No significant changes were seen in the sphingosine response $(p=0.1)$. CER (18: / 22: 0), CER (18: 1/24: 0) / (18: 0/24: 1), and CER (18: 1/24: 1) were the ceramide subclasses with the highest peak response; whereas SM (18:1/16:0) and SM (42: 2) were the highest among the sphingomyelin species. Cholesterol esters increased significantly at T1. CE (18: 1), (18: 2), and (20:4) were those that showed the most significant increase. All these results by subgroups generated a significant increase in the total lipid average responses between both studied periods. All the results analyzed by groups are summarized in Figure 1.

\section{Discussion}

This study aimed to evaluate the change in the main lipid class and subclass profiles after starting CAPD. Dyslipidemia is an important cardiovascular risk factor in the general population, as shown in numerous clinical studies ${ }^{21,22}$. The SHARP clinical study revealed these same beneficial effects in the NDD-CKD population but could not demonstrate them in those on dialysis ${ }^{23}$; a lack of benefit also reported in the AURORA $^{24}$ and $4 \mathrm{D}$ trials $^{25}$. Some studies have detected a paradoxical epidemiological relationship with higher mortality reported in those patients with the lowest cholesterol levels ${ }^{26}$; however, this could be attributed to malnourished dialysis patients with shorter life expectancy and multiple comorbidities ${ }^{27}$. There is scarce data on the effect of dyslipidemia in patients in PD, and the actual value of classical lipid biomarkers is unclear.

Our LC-QTOF/MS-based lipidomics approach to study the effect of starting renal replacement therapy with CAPD in patients with CKD shows that total FFAs increased after six months on this technique. Within this group of lipids, FA 16:1 (palmitoleic acid) and FA 18:0 (stearic acid) were the ones that increased the most. FFAs are among the most important lipid classes and have many biological functions, regulating physiological pro-inflammatory response, dilating vessels, and signal molecules to regulate the nervous system ${ }^{28,29}$. A study by Friedman et al. demonstrated that FFAs levels are increased in the plasma of CKD patients; moreover, they have reported that higher levels of saturated fatty acids in serum correlate with sudden cardiac death in patients starting hemodialysis ${ }^{30}$. Likewise, monounsaturated fatty acids are the products of Stearoyl-CoA desaturase (SCD) catalyzed reactions and act as substrates to synthesize phospholipids and triglycerides ${ }^{31}$. SCD is a critical enzyme in fatty acid metabolism and an important enzyme that interacted with different hormones, such as insulin or leptin ${ }^{32-33}$. The activity of SCD is very high in patients with cardiovascular disease, hypertension, and diabetes $^{34-35}$; moreover, the ratios of FFA 16:1 / FFA 16:0 and FFA 18:1 / FFA 18:0 levels are considered as reference values to estimate the activity of $S D^{36}$. Our study found that the ratios of FFA 16: 1 / FFA 
16: 0 significantly increased from T0 to T1; on the contrary, this did not happen with the ratios of FFA 18: 1 / FFA 18: 0.

Our results show that $\mathrm{PC}$ and $\mathrm{PE}$ increased significantly at $\mathrm{T} 1$, but there were no changes in LysoPC levels, which is an essential component of oxidized LDL and accumulates in atherosclerotic lesions in experimental animals ${ }^{37}$. Therefore, it has been considered as a mediator of the atherogenic effect of oxidized LDL. LysoPCs are generated from PCs by the enzymatic activity of lecithin: cholesterol acyltransferase (LCAT). LCAT deficiency and low LysoPC levels have been reported and in HD patients, where low LysoPC levels were associated with increased cardiovascular risk compared with a control group ${ }^{38}$. To our knowledge, this is the first study to analyze this group of lipids in PD patients.

Alshehry et al. studies showed that sphingolipids, phospholipids, cholesteryl esters, and glycerolipids were associated with future cardiovascular events and cardiovascular death in patients with type 2 diabetes $^{39}$. Elevated sphingomyelin levels have been associated with a risk factor for coronary heart disease and with lower survival of the PD technique ${ }^{40}$. To our knowledge, it is the first study to find increased levels of glycerolipid, sphingolipids, and cholesterol esters six months after initiating CAPD.

Our study has some limitations since it involves young patients with a short follow-up period, and unlike others that cross-compare two different groups, it has a short follow up period that made the measurement of cardiovascular events inappropriate, and as most CKD lipidomic profile studies, we only analyzed a small subset of 39 patients. On the other hand, our study's strength is that it involves a single cohort of patients followed for six months with blood samples taken at baseline and after a six-month exposure to a significant glucose load through the dialysate solutions without changes in the treatment during follow-up.

In conclusion, our results show that although the traditionally used lipid biomarkers may not change after the start of PD, the alterations in the lipidomic profile found suggest an increased cardiovascular risk in this population.

\section{Declarations}

\section{Acknowledgments}

We want to thank the selflessly included patients, peritoneal dialysis nurse team and analytical unit platform staff for their inestimable help on this study's execution.

\section{Authors' Contributions}

J.H.L. and J.H.J. designed the study; J.H.J., M.R.M., J.H.L. and L.C.L.R. carried out experiments; M.R.M., J.J.B.M., L.C.L.R., I.V.B. and M.A.K. analyzed the data; P.S.P., A.S.O. and R.D.S. made the figures; M.R.M., L.C.L.R., D.R-E., and J.J.B.M drafted and revised the paper; all authors approved the final version of the manuscript. 
The authors declare no competing interests. This work has received no public or private funds.

\section{References}

1. Quehenberger O, Dennis EA. The human plasma lipidome. N Engl J Med. 2011;365(19):1812-1823.

2. Nicolson GL, Ash ME. Lipid Replacement Therapy: a natural medicine approach to replacing damaged lipids in cellular membranes and organelles and restoring function. Biochim Biophys Acta. 2014 Jun;1838(6):1657-79. doi: 10.1016/j.bbamem.2013.11.010. Epub 2013 Nov 21. PMID: 24269541.

3. Sarnak MJ, Levey AS, Schoolwerth AC, Coresh J, Culleton B, Hamm LL, McCullough PA, Kasiske BL, Kelepouris E, Klag MJ, Parfrey P, Pfeffer M, Raij L, Spinosa DJ, Wilson PW; American Heart Association Councils on Kidney in Cardiovascular Disease, High Blood Pressure Research, Clinical Cardiology, and Epidemiology and Prevention. Kidney disease as a risk factor for development of cardiovascular disease: a statement from the American Heart Association Councils on Kidney in Cardiovascular Disease, High Blood Pressure Research, Clinical Cardiology, and Epidemiology and Prevention. Hypertension. 2003 Nov;42(5):1050-65. doi: 10.1161/01.HYP.0000102971.85504.7c. PMID: 14604997.

4. Schiffrin EL, Lipman ML, Mann JF. Chronic kidney disease: effects on the cardiovascular system. Circulation. 2007 Jul 3;116(1):85-97. doi: 10.1161/CIRCULATIONAHA.106.678342. PMID: 17606856.

5. Vaziri ND. Dyslipidemia of chronic renal failure: the nature, mechanisms, and potential consequences. Am J Physiol Renal Physiol. 2006 Feb;290(2):F262-72. doi: 10.1152/ajprenal.00099.2005. PMID: 16403839.

6. Vaziri ND, Norris K. Lipid disorders and their relevance to outcomes in chronic kidney disease. Blood Purif. 2011;31(1-3):189-96. doi: 10.1159/000321845. Epub 2011 Jan 10. PMID: 21228589.

7. Mikolasevic I, Žutelija M, Mavrinac V, Orlic L. Dyslipidemia in patients with chronic kidney disease: etiology and management. Int J Nephrol Renovasc Dis. 2017 Feb 7;10:35-45. doi: 10.2147/IJNRD.S101808. PMID: 28223836; PMCID: PMC5304971.

8. Guo Q, Lin J, Li J, Yi C, Mao H, Yang X, Yu X. The Effect of Fluid Overload on Clinical Outcome in Southern Chinese Patients Undergoing Continuous Ambulatory Peritoneal Dialysis. Perit Dial Int. 2015 Dec;35(7):691-702. doi: 10.3747/pdi.2014.00008. Epub 2015 Jul 7. PMID: 26152580; PMCID: PMC4690624.

9. Rincón Bello A, Bucalo L, Abad Estébanez S, Vega Martínez A, Barraca Núñez D, Yuste Lozano C, Pérez de José A, López-Gómez JM. Fat tissue and inflammation in patients undergoing peritoneal dialysis. Clin Kidney J. 2016 Jun;9(3):374-80. doi: 10.1093/ckj/sfw007. Epub 2016 Mar 24. PMID: 27274820; PMCID: PMC4886903.

10. LaRosa JC, Grundy SM, Waters DD, Shear C, Barter P, Fruchart JC, Gotto AM, Greten H, Kastelein JJ, Shepherd J, Wenger NK; Treating to New Targets (TNT) Investigators. Intensive lipid lowering with 
atorvastatin in patients with stable coronary disease. N Engl J Med. 2005 Apr 7;352(14):1425-35. doi: 10.1056/NEJMoa050461. Epub 2005 Mar 8. PMID: 15755765.

11. Baigent C, Landray MJ, Reith C, Emberson J, Wheeler DC, Tomson C, Wanner C, Krane V, Cass A, Craig J, Neal B, Jiang L, Hooi LS, Levin A, Agodoa L, Gaziano M, Kasiske B, Walker R, Massy ZA, FeldtRasmussen B, Krairittichai U, Ophascharoensuk V, Fellström B, Holdaas H, Tesar V, Wiecek A, Grobbee D, de Zeeuw D, Grönhagen-Riska C, Dasgupta T, Lewis D, Herrington W, Mafham M, Majoni W, Wallendszus K, Grimm R, Pedersen T, Tobert J, Armitage J, Baxter A, Bray C, Chen Y, Chen Z, Hill M, Knott C, Parish S, Simpson D, Sleight P, Young A, Collins R; SHARP Investigators. The effects of lowering LDL cholesterol with simvastatin plus ezetimibe in patients with chronic kidney disease (Study of Heart and Renal Protection): a randomised placebo-controlled trial. Lancet. 2011 Jun 25;377(9784):2181-92. doi: 10.1016/S0140-6736(11)60739-3. Epub 2011 Jun 12. PMID: 21663949; PMCID: PMC3145073.

12. Han X, Gross RW. Global analyses of cellular lipidomes directly from crude extracts of biological samples by ESI mass spectrometry: a bridge to lipidomics. J Lipid Res. 2003 Jun;44(6):1071-9. doi: 10.1194/jlr.R300004-JLR200. Epub 2003 Apr 1. PMID: 12671038.

13. Loomba R, Quehenberger O, Armando A, Dennis EA. Polyunsaturated fatty acid metabolites as novel lipidomic biomarkers for noninvasive diagnosis of nonalcoholic steatohepatitis. J Lipid Res. 2015 Jan;56(1):185-92. doi: 10.1194/jlr.P055640. Epub 2014 Nov 17. PMID: 25404585; PMCID: PMC4274066.

14. Tam VC, Quehenberger O, Oshansky CM, Suen R, Armando AM, Treuting PM, Thomas PG, Dennis EA, Aderem A. Lipidomic profiling of influenza infection identifies mediators that induce and resolve inflammation. Cell. 2013 Jul 3;154(1):213-27. doi: 10.1016/j.cell.2013.05.052. PMID: 23827684; PMCID: PMC3753192.

15. Ji J, Kline AE, Amoscato A, Samhan-Arias AK, Sparvero LJ, Tyurin VA, Tyurina YY, Fink B, Manole MD, Puccio AM, Okonkwo DO, Cheng JP, Alexander H, Clark RS, Kochanek PM, Wipf P, Kagan VE, Bayır H. Lipidomics identifies cardiolipin oxidation as a mitochondrial target for redox therapy of brain injury. Nat Neurosci. 2012 Oct;15(10):1407-13. doi: 10.1038/nn.3195. Epub 2012 Aug 26. PMID: $22922784 ;$ PMCID: PMC3697869.

16. Reis A, Rudnitskaya A, Chariyavilaskul P, Dhaun N, Melville V, Goddard J, Webb DJ, Pitt AR, Spickett CM. Top-down lipidomics of low density lipoprotein reveal altered lipid profiles in advanced chronic kidney disease. J Lipid Res. 2015 Feb;56(2):413-22. doi: 10.1194/jlr.M055624. Epub 2014 Nov 25. PMID: 25424003; PMCID: PMC4306694.

17. Zhao YY. Metabolomics in chronic kidney disease. Clin Chim Acta. 2013 Jun 25;422:59-69. doi: 10.1016/j.cca.2013.03.033. Epub 2013 Apr 6. PMID: 23570820.

18. Zhao YY, Wu SP, Liu S, Zhang Y, Lin RC. Ultra-performance liquid chromatography-mass spectrometry as a sensitive and powerful technology in lipidomic applications. Chem Biol Interact. 2014 Sep 5;220:181-92. doi: 10.1016/j.cbi.2014.06.029. Epub 2014 Jul 9. PMID: 25014415. 
19. Wanner C, Tonelli M; Kidney Disease: Improving Global Outcomes Lipid Guideline Development Work Group Members. KDIGO Clinical Practice Guideline for Lipid Management in CKD: summary of recommendation statements and clinical approach to the patient. Kidney Int. 2014 Jun;85(6):13039. doi: 10.1038/ki.2014.31. Epub 2014 Feb 19. PMID: 24552851.

20. Alcoriza-Balaguer MI, García-Cañaveras JC, López A, Conde I, Juan O, Carretero J, Lahoz A. LipidMS: An R Package for Lipid Annotation in Untargeted Liquid Chromatography-Data Independent Acquisition-Mass Spectrometry Lipidomics. Anal Chem. 2019 Jan 2;91(1):836-845. doi: 10.1021/acs.analchem.8b03409. Epub 2018 Dec 13. PMID: 30500173.

21. Sarnak MJ, Levey AS, Schoolwerth AC, Coresh J, Culleton B, Hamm LL, McCullough PA, Kasiske BL, Kelepouris E, Klag MJ, Parfrey P, Pfeffer M, Raij L, Spinosa DJ, Wilson PW; American Heart Association Councils on Kidney in Cardiovascular Disease, High Blood Pressure Research, Clinical Cardiology, and Epidemiology and Prevention. Kidney disease as a risk factor for development of cardiovascular disease: a statement from the American Heart Association Councils on Kidney in Cardiovascular Disease, High Blood Pressure Research, Clinical Cardiology, and Epidemiology and Prevention. Hypertension. 2003 Nov;42(5):1050-65. doi: 10.1161/01.HYP.0000102971.85504.7c. PMID: 14604997.

22. Cholesterol Treatment Trialists' (CTT) Collaboration, Baigent C, Blackwell L, Emberson J, Holland LE, Reith C, Bhala N, Peto R, Barnes EH, Keech A, Simes J, Collins R. Efficacy and safety of more intensive lowering of LDL cholesterol: a meta-analysis of data from 170,000 participants in 26 randomised trials. Lancet. 2010 Nov 13;376(9753):1670-81. doi: 10.1016/S0140-6736(10)61350-5. Epub 2010 Nov 8. PMID: 21067804; PMCID: PMC2988224.

23. Sharp Collaborative Group. Study of Heart and Renal Protection (SHARP): randomized trial to assess the effects of lowering low-density lipoprotein cholesterol among 9,438 patients with chronic kidney disease. Am Heart J. 2010 Nov;160(5):785-794.e10. doi: 10.1016/j.ahj.2010.08.012. Epub 2010 Sep 18. PMID: 21095263.

24. Fellström, B., Zannad, F., Schmieder, R., Holdaas, H., Jardine, A., Rose, H., Wilpshaar, W., \& AURORA Study Group (2005). Effect of rosuvastatin on outcomes in chronic haemodialysis patients - design and rationale of the AURORA study. Current controlled trials in cardiovascular medicine, 6(1), 9. https://doi.org/10.1186/1468-6708-6-9

25. Wanner C, Krane V, März W, Olschewski M, Asmus HG, Krämer W, Kühn KW, Kütemeyer H, Mann JF, Ruf G, Ritz E; Deutsche Diabetes-Dialyse-Studie (4D) Study Group. Randomized controlled trial on the efficacy and safety of atorvastatin in patients with type 2 diabetes on hemodialysis (4D study): demographic and baseline characteristics. Kidney Blood Press Res. 2004;27(4):259-66. doi: 10.1159/000080241. Epub 2004 Aug 16. PMID: 15316128.

26. Schiffrin EL, Lipman ML, Mann JF. Chronic kidney disease: effects on the cardiovascular system. Circulation. 2007 Jul 3;116(1):85-97. doi: 10.1161/CIRCULATIONAHA.106.678342. PMID: 17606856.

27. Liu Y, Coresh J, Eustace JA, Longenecker JC, Jaar B, Fink NE, Tracy RP, Powe NR, Klag MJ. Association between cholesterol level and mortality in dialysis patients: role of inflammation and malnutrition. JAMA. 2004 Jan 28;291(4):451-9. doi: 10.1001/jama.291.4.451. PMID: 14747502. 
28. Keane, W. F.,J. E.Tomassini, and D. R.Neff. 2013. Lipid abnormalities in patients with chronic kidney disease: implications for the pathophysiology of atherosclerosis.J. Atheroscler. Thromb.20:123-13.

29. Varga, Z.; Karpati, I.; Paragh, G. Relative abundance of some free fatty acids in plasma of uremic patients: Relationship between Fatty Acids, Lipid Parameters, and disease. Nephron 1997, 77, 417421.

30. Friedman AN, Yu Z, Denski C, Tamez H, Wenger J, Thadhani R, Li Y, Watkins B. Fatty acids and other risk factors for sudden cardiac death in patients starting hemodialysis. Am J Nephrol. 2013;38(1):12-8. doi: 10.1159/000351764. Epub 2013 Jun 25. PMID: 23816975; PMCID: PMC3856432.

31. Miyazaki, Makoto; Ntambi, James M. (2003-02-01). "Role of stearoyl-coenzyme A desaturase in lipid metabolism". Prostaglandins, Leukotrienes, and Essential Fatty Acids. 68 (2): 113-121.

32. Ntambi JM. Regulation of stearoyl-CoA desaturase by polyunsaturated fatty acids and cholesterol. J Lipid Res. 1999 Sep;40(9):1549-58. PMID: 10484602.

33. Ntambi JM, Miyazaki M, Stoehr JP, Lan H, Kendziorski CM, Yandell BS, Song Y, Cohen P, Friedman JM, Attie AD. Loss of stearoyl-CoA desaturase-1 function protects mice against adiposity. Proc Natl Acad Sci U S A. 2002 Aug 20;99(17):11482-6. doi: 10.1073/pnas.132384699. Epub 2002 Aug 12. PMID: 12177411; PMCID: PMC123282.

34. Mar-Heyming R, Miyazaki M, Weissglas-Volkov D, et al. Association of stearoyl-CoA desaturase 1 activity with familial combined hyperlipidemia. Arteriosclerosis, Thrombosis, and Vascular Biology. 2008 Jun;28(6):1193-1199. DOI: 10.1161/atvbaha.107.160150. PMID: 18340007; PMCID: PMC2758768.

35. Brown, J. M.; Chung, S.; Sawyer, J. K.; Degirolamo, C.; Alger, H. M.; Nguyen, T. M.; Zhu, X.; Duong, M. N.; Brown, A. L.; Lord, C.; Shah, R.; Davis, M. A.; Kelley, K.; Wilson, M. D.; Madenspacher, J.; Fessler, M. B.;Parks, J. S.; Rudel, L. L. Combined therapy of dietary fish oil and stearoyl-CoA desaturase 1 inhibition prevents the metabolic syndrome and atherosclerosis. Arterioscler Thromb Vasc Biol 2010,30 (1), 24-30.

36. Attie AD, Krauss RM, Gray-Keller MP, Brownlie A, Miyazaki M, Kastelein JJ, Lusis AJ, Stalenhoef AF, Stoehr JP, Hayden MR, Ntambi JM. Relationship between stearoyl-CoA desaturase activity and plasma triglycerides in human and mouse hypertriglyceridemia. J Lipid Res. 2002 Nov;43(11):1899907. doi: 10.1194/jIr.m200189-jIr200. PMID: 12401889.

37. Mclntyre TM, Zimmerman GA, Prescott SM. Biologically active oxidized phospholipids. J Biol Chem. 1999 Sep 3;274(36):25189-92. doi: 10.1074/jbc.274.36.25189. PMID: 10464236.

38. Lee, Y.-K.; Lee, D. H.; Kim, J. K.; Park, M.-J.; Yan, J.-J.; Song, D.-K.; Vaziri, N. D.; Noh, J.-W Lysophosphatidylcholine, Oxidized Low-Density Lipoprotein and Cardiovascular Disease in Korean Hemodialysis Patients: Analysis at 5 Years of Follow-up. J Korean Med Sci 2013, 28, 268-273.

39. Alshehry ZH, Mundra PA, Barlow CK, Mellett NA, Wong G, McConville MJ, Simes J, Tonkin AM, Sullivan DR, Barnes EH, Nestel PJ, Kingwell BA, Marre M, Neal B, Poulter NR, Rodgers A, Williams B, Zoungas S, Hillis GS, Chalmers J, Woodward M, Meikle PJ. Plasma Lipidomic Profiles Improve on 
Traditional Risk Factors for the Prediction of Cardiovascular Events in Type 2 Diabetes Mellitus. Circulation. 2016 Nov 22;134(21):1637-1650. doi: 10.1161/CIRCULATIONAHA.116.023233. Epub 2016 Oct 18. PMID: 27756783.

40. Schlitt, A., Blankenberg, S.,Yan, D., von Gizycki, H., Buerke, M., Werdan, K., Jiang, X. Ch. (2006). Further evaluation of plasma sphingomyelin levels as a risk factor for coronary artery disease. Nutr Metab (Lond), 3, 5.

\section{Figures}

Lipids families
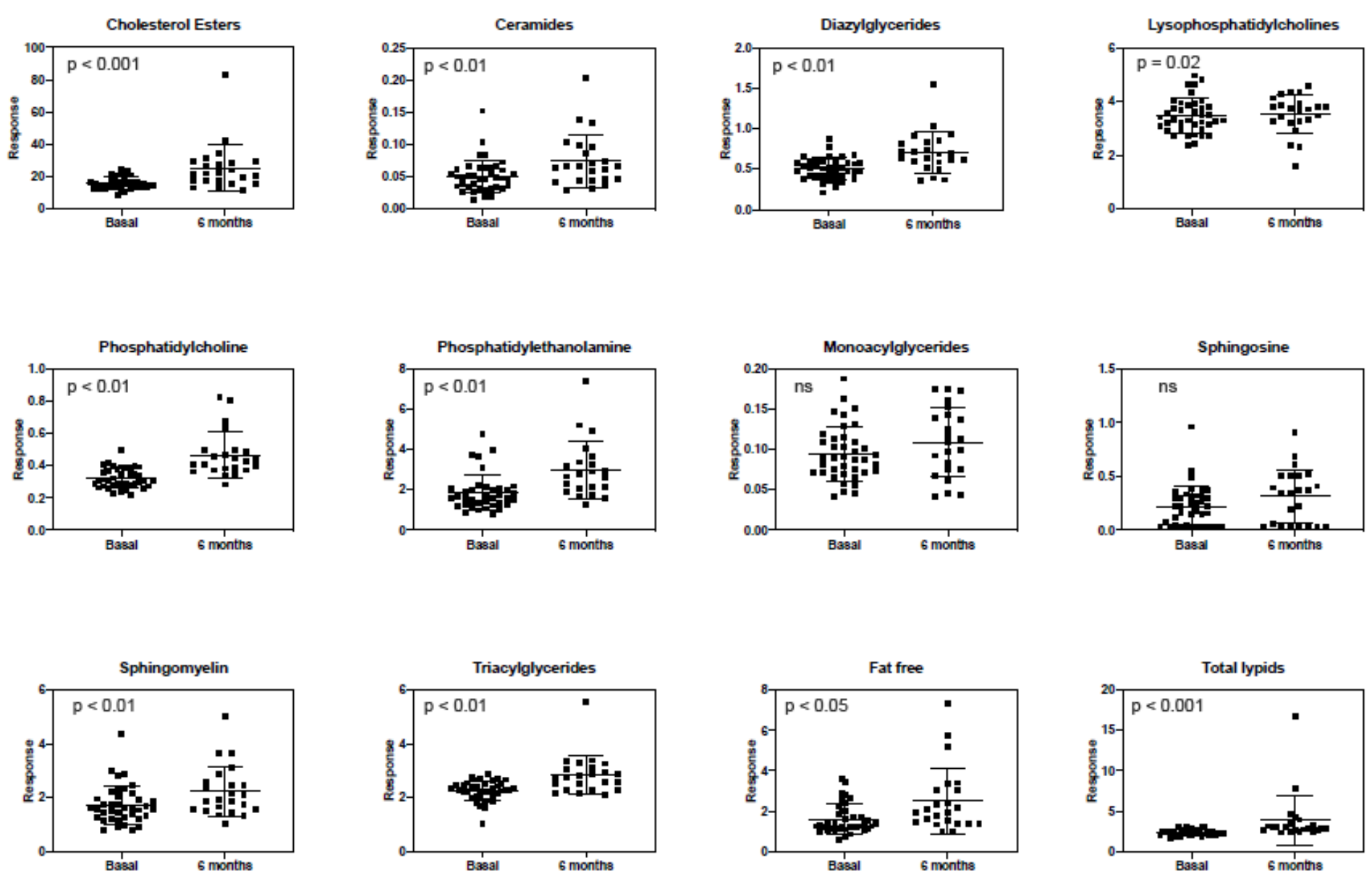

\section{Figure 1}

Comparison of lipid subclass levels between the measurement time at the beginning of CAPD and 6 months after 